\title{
Características sociodemográficas e acadêmicas de estudantes de enfermagem em formação profissional
}

\author{
Socio-demographic and academic \\ characteristics of nursing students in \\ professional training
}

\author{
Mari Saho' ${ }^{1}$ \\ Georgina Almeida Lomanto ${ }^{2}$ (1) \\ Isabel Cristina de Barros Salviano ${ }^{3}$ (1)
}

\section{Erenice Santos Reis ${ }^{4}$ (1) Karla Ferraz dos Anjos ${ }^{5}$ (1) Darci de Oliveira Santa Rosa ${ }^{6}$ (1)}

${ }^{1}$ Autora para correspondência. Centro UNIVERSO Salvador (Salvador). Bahia, Brasil. sahomarijk@gmail.com ${ }^{2}$ Associação Salgado de Oliveira de Educação e Cultura (Salvador), Bahia, Brasil. georginalomanto@hotmail.com ${ }^{3}$ Centro UNIVERSO Salvador (Salvador). Bahia, Brasil. isabel.salviano@gmail.com 4-6Universidade Federal da Bahia (Salvador). Bahia, Brasil. erereis@yahoo.com.br karla.ferraz@hotmail.com, darcisantarosa@gmail.com

RESUMO | OBJETIVO: Descrever as características sociodemográficas e acadêmicas de estudantes de enfermagem de uma instituição de ensino superior sobre sua formação profissional. MÉTODO: Estudo descritivo, quantitativo, realizado com 197 estudantes do curso de graduação em enfermagem, matriculados em todos os períodos do curso. Os dados foram coletados por meio de um questionário fechado autopreenchido e aplicado no período de maio a agosto de 2017. RESULTADOS: Evidenciouse entre os estudantes de enfermagem o predomínio do sexo feminino $(82,2 \%)$, solteiros $(76,1 \%)$, com inserção no mercado de trabalho formal $(49,7 \%)$, possuem filhos $(44,7 \%)$, residem distantes da unidade de ensino (69\%), utilizam transporte coletivo para deslocar para a IES $(82,7 \%)$ e faz uso da internet como principal recurso para os estudos $(91,4 \%)$. Entre as áreas de atuação almejadas durante a formação, tem-se a de assistência e da enfermagem obstétrica. CONCLUSÃO: Verificou-se possíveis contribuições à estruturação do projeto pedagógico do curso, uma vez que o estudo traz elementos que caracterizam o perfil e algumas demandas acadêmicas dos estudantes que frequentam o curso de graduação em enfermagem de uma instituição de ensino.

DESCRITORES: Educação em Enfermagem. Educação superior. Estudantes de Enfermagem.

\begin{abstract}
OBJECTIVE: To describe nursing students' sociodemographic and academic characteristics from a higher education institution regarding their professional training. METHOD: Descriptive, quantitative study, carried out with 197 undergraduate nursing students enrolled in all course periods. Data were collected through a closed, selfadministered questionnaire applied from May to August 2017. RESULTS: Among nursing students, there was a predominance of females (82.2\%), singles (76.1\%), with insertion in the formal labor market $(49.7 \%)$, have children $(44.7 \%)$, live far from the teaching unit $(69 \%)$, use public transport to travel to the HEI $(82.7 \%)$ and uses the internet as the main resource for studies (91.4\%). Among the areas of action desired during training, there is assistance and obstetric nursing. CONCLUSION: Possible contributions to the structuring of the pedagogical project of the course were verified since the study brings elements that characterize the profile and some academic demands of students who attend the undergraduate nursing course of an educational institution.
\end{abstract}

DESCRIPTORS: Nursing Education. College education. Nursing students. 


\section{Introdução}

O ensino de Enfermagem desenvolvido nas propostas curriculares da formação de enfermeiros tem sofrido transformação nas últimas décadas, principalmente a partir da nova Lei de Diretrizes e Bases da Educação Nacional (LDB /1996). As mudanças trouxeram novas responsabilidades para as Instituições de Ensino Superior (IES), docentes, discentes e sociedade em geral, por permitir a formulação de diferentes perfis profissionais a partir da vocação de cada curso/ escola, para melhor adaptação ao mundo de trabaIho, uma vez que as instituições de ensino obtiveram autonomia para definir parte considerável de seus currículos plenos.

Para o curso superior de Enfermagem, a Resolução do Conselho Nacional de Educação (CNE/CFE N03/2001), que define as Diretrizes Curriculares Nacionais para o Curso de Graduação em Enfermagem (DCENF), trouxe de concreto as mudanças propostas no processo ensino e aprendizagem para a formação dos enfermeiros com a implementação de um Projeto Pedagógico do Curso (PPC) alinhado aos princípios e diretrizes do Sistema Único de Saúde (SUS). ${ }^{1}$

As DCENF1, dentre outras, descrevem o perfil esperado do egresso, a partir de formação generalista ${ }^{1}$, o que precisa ser considerado pelas IES. Em relação aos cursos de graduação em Enfermagem, a tendência de privatização do ensino se acentuou nos primeiros anos de 2000 , quando $63,6 \%$ dos cursos de Enfermagem existentes no Brasil pertenciam à rede privada de ensino. Vale assinalar, ainda, que este processo de privatização dos cursos obteve um crescimento de 75,7\% no período de 2010 a 2013. Enquanto o de graduados de Enfermagem na rede pública, que no período de 1990 a 1999 representava $59,1 \%$ do total, decresceu para $19 \%$ no período de 2010 a 2013 . 2

Quando se observa a distribuição territorial dos cursos de Enfermagem e a atuação dos profissionais, nota-se uma assimetria regional no país. A exemplo, a existência acentuada dos cursos de graduação e pós-graduação em grandes regiões, como Sul e Sudeste, em detrimento de outras regiões, como Norte, Nordeste e Centro-oeste. A permanência dos profissionais de Enfermagem em capitais e grandes centros urbanos em Estados é uma tendência.?
Observa-se, ainda, o aumento médio da faixa etária dos estudantes de enfermagem e aqueles já inseridos no mercado de trabalho de nível médio que almejam uma qualificação profissional através do acesso ao ensino superior e que escolhem o curso de Enfermagem como profissão.? Deve-se salientar que em paralelo às mudanças que vêm ocorrendo no perfil de estudantes que buscam a qualificação profissional de nível superior, a proposta de formar enfermeiro generalista, humanista, crítico e reflexivo, dotado de rigor científico e intelectual baseado em princípios éticos contidos nas DCENF exige uma mudança na construção de um projeto pedagógico do curso (PPC) de Enfermagem que contemple transformações no processo de ensino e aprendizagem. ${ }^{3-4}$

Na proposta das DCENF de 2001, a educação é compreendida como um processo permanente de aprendizagem que se inicia na graduação, estende-se por toda atuação profissional, mantém uma relação de parceria entre as IES e os serviços de saúde/comunidade e outras entidades civis. ${ }^{1}$ Tão logo, é nessa perspectiva de formação, junto à comunidade, que é preciso construir o PPC, de forma coletiva, com foco no estudante como sujeito ativo do processo de aprendizagem e o professor como mediador desse processo sob a construção de um currículo de forma integrada, o qual privilegia a interdisciplinaridade do conhecimento para a formação da pessoa competente profissionalmente, dotada de espírito cidadão e de solidariedade. ${ }^{4}$

Nesse cenário, o ensino centrado no professor se desloca para a ênfase na aprendizagem do estudante por meio de reconfiguração dos saberes, através da diminuição das clássicas dualidades: saber científico/ saber popular, ciência/cultura, educação/trabalho. Busca-se uma reorganização da relação teoria/prática e rompe-se com a visão tradicional de que a teoria precede a prática. De igual maneira, desloca-se para a mediação entre as subjetividades dos envolvidos e o conhecimento, contempla-se a dimensão das relações, do respeito mútuo, dos laços que se estabelecem entre os atores, dando-se ênfase à participação ativa dos estudantes nas decisões pedagógicas em prol da produção pessoal, original e criativa. ${ }^{3}$

Portanto, os eixos orientadores de políticas públicas de educação atual vislumbram a formação do enfermeiro 
generalista, capaz de atuar na realidade da atenção à saúde, que é complexa e dinâmica. A prioridade gira em torno de atenção básica à saúde (ABS), reflexão crítica dos processos que envolvem a saúde, doença e cuidado individual e coletivo da população. Esta reflexão abrange o processo de trabalho na produção do cuidado, utiliza-se o referencial pedagógico da competência e interdisciplinaridade que abrange os temas como ética, trabalho em equipe multidisciplinar e humanização da assistência à saúde. $\frac{5}{}$

No processo de aprendizagem, é fundamental a aproximação do professor aos estudantes do curso, conhecendo seu perfil, seu projeto de vida, sua especificidade pessoal, histórica e social. Com essa aproximação, é possível conhecer o estudante, seus interesses pessoais e sociais em relação a sua formação profissional enfermeiro. ${ }^{5}$ Justifica-se, assim, a realização do estudo proposto, conhecendo o perfil dos estudantes, sobre as características sóciodemográficas e seus anseios em relação ao ensino no âmbito de um curso de enfermagem, para contribuir na elaboração de um projeto de curso mais condizente com real demanda dos estudantes.

Neste contexto, o estudo teve as seguintes questões de pesquisa: que características sociodemográficas apresentam os estudantes de enfermagem que frequentam uma IES? Quais são seus anseios acadêmicos do estudante em relação ao curso de formação do profissional enfermeiro?

Estudo tem como objetivo descrever as características sociodemográficas e acadêmicas de estudantes de enfermagem de uma instituição de ensino superior sobre sua formação profissional.

\section{Método}

Estudo descritivo, de natureza quantitativa, desenvolvido em uma IES no Município de Salvador-Bahia. A população de estudo foi formada por estudantes de enfermagem de todos os períodos que compõem um curso de graduação, em uma instituição de ensino superior no Município de Salvador-Bahia, composta por 197 pesquisados, correspondendo a $70 \%$ do total de estudantes matriculados no semestre 2017.1. Houve a participação de um pequeno número de estudantes dos dois últimos períodos de enfermagem devido à dificuldade em reuni-los, e, consequentemente, adesão destes, por estarem matriculados em componentes curriculares práticos e estarem em campos de práticas.

Como critérios de inclusão, foram considerados estudantes de enfermagem regularmente matriculados, que frequentavam as aulas no período de coleta de dados (maio a agosto de 2017), com idade igual ou superior a 18 anos. Excluídos da amostra, os estudantes que temporariamente estavam afastados da sala de aula por motivos de licença médica e problemas familiares e/ou pessoais.

Todos os estudantes presentes em sala de aula, no dia e horário da coleta dos dados, eram convidados para participar da pesquisa. Após aceite, era disponibilizado o Termo de Consentimento Livre e Esclarecido. Em seguida, o questionário contendo 22 questões, com questionamentos sobre características sociodemográficas, acadêmicas e profissionais foi entregue e preenchido pelos estudantes. Ressalta-se que o instrumento permitiu ao participante do estudo responder mais de uma alternativa em cada pergunta apresentada, a exemplo das experiências práticas, funções almejadas na vida profissional. No total, foram 280 estudantes elegíveis e, ao final, 197 participantes do estudo.

A coleta de dados foi realizada no período de maio a agosto de 2017, e se deu por meio de entrevista com aplicação de um questionário no período pós aula, combinado com antecipação com estudantes e professores responsáveis pela pesquisa, e foi preenchido pelo próprio entrevistado e entregue a uma das pesquisadoras.

Para caracterizar os estudantes que frequentam um curso de graduação em enfermagem em uma IES particular, foi necessário construir um instrumento tipo questionário de perguntas fechadas que permitiu coletar os dados que representasse a realidade dos mesmos. Este instrumento continha as seguintes variáveis: dados sociodemográficos (sexo, idade, ocupação, inserção no mercado de trabalho, procedência em termos de local de moradia), dados acadêmicos (tempo dedicado aos estudos e as técnicas pedagógicas valorizadas) e expectativas profissionais (área de atuação futura e as funções almejadas ao exercer a profissão de enfermagem). 
Os dados coletados foram armazenados em planilha Excel 7. Para a análise, aplicou-se a estatística descritiva, apresentando-se em frequências absoluta e relativa.

Quanto aos aspectos éticos do estudo, o mesmo segue os preceitos da Resolução 466/12 do Conselho Nacional de Saúde, aprovado pelo Comitê de Ética em Pesquisa da Escola de Enfermagem da UFBA, com CAAE: 62976316.50000.5531, e parecer de aprovação concedido pelo número 1.977.500, em 22 de março de 2017.

\section{Resultados}

Dos 197 estudantes, em sua maioria, representando 82,2\% (162) foram do sexo feminino, 43,1\% (85) com faixa etária de 20 a 30 anos de idade, 76,1\% (150) solteiro e 54,8\%(108) não têm filhos, 49,7\% (98) possuem vínculo empregatício, sendo que entre estes, 18,8\% (37) na área de saúde; 69,5\% (137) possuem casa própria, 82,7\% (163) se locomoviam de transporte coletivo, 31\% (61) residiam em bairros próximos à faculdade, conforme explicita a tabela 1.

Tabela 1. Características sociodemgráficas de estudantes do curso de graduação em enfermagem, Salvador, Bahia, Brasil. 2017

\begin{tabular}{|c|c|c|}
\hline Variáveis & $n$ & $\%$ \\
\hline \multicolumn{3}{|l|}{ Sexo } \\
\hline Feminino & 162 & 82,2 \\
\hline Masculino & 35 & 17,8 \\
\hline \multicolumn{3}{|l|}{ Faixa etária (em anos) } \\
\hline$<20$ anos & 34 & 17,3 \\
\hline $20-30$ anos & 85 & 43,1 \\
\hline $30-40$ anos & 46 & 23,6 \\
\hline 40-50 anos & 27 & 13,7 \\
\hline$>50$ anos & 4 & 2,0 \\
\hline \multicolumn{3}{|l|}{ Estado civil } \\
\hline Solteiro & 150 & 76,1 \\
\hline Casado & 36 & 18,3 \\
\hline Divorciado & 8 & 4,1 \\
\hline Viúvo & 2 & 2,0 \\
\hline \multicolumn{3}{|l|}{ Ter filhos } \\
\hline Não & 108 & 54,8 \\
\hline Sim & 88 & 45,2 \\
\hline \multicolumn{3}{|l|}{ Natureza da ocupação } \\
\hline Estudante apenas & 112 & 56,9 \\
\hline Assistência à saúde & 37 & 18,8 \\
\hline Administrativa & 33 & 16,8 \\
\hline Comércio & 5 & 2,5 \\
\hline Serviços gerais & 5 & 2,5 \\
\hline Ensino (professor) & 4 & 2,0 \\
\hline \multicolumn{3}{|l|}{ Moradia } \\
\hline Própria & 137 & 69,5 \\
\hline Alugada & 35 & 17,8 \\
\hline Cedida & 3 & 1,5 \\
\hline Outras & 22 & 11,2 \\
\hline \multicolumn{3}{|l|}{ Transporte de locomoção ao curso } \\
\hline Coletivo & 163 & 82,7 \\
\hline Carro próprio & 26 & 13,2 \\
\hline Caminhada & 7 & 3,6 \\
\hline \multicolumn{3}{|l|}{ Local de moradia } \\
\hline Bairros distantes ao curso & 125 & 63,5 \\
\hline Bairros próximos ao curso & 61 & 31,0 \\
\hline Região Metropolitana de SSA/Interior & 11 & 5,6 \\
\hline
\end{tabular}


No que se refere ao tempo dedicado aos estudos fora da sala de aula, foi em torno de 3 a 4 horas diárias para $36 \%$ (71) dos entrevistados, e acima de 4 horas de estudo para 32,5\% (64). Em relação à preferência do local para estudos, o domicílio, com 85,3\% (168), teve maior percentual. O material frequentemente utilizado para os estudos provém da internet com 91,4\% (180), com preferência do estudo individual 72,9\% (143), seguida do estudo em grupo $31 \%$ (61). As técnicas de ensino vivenciadas no curso citadas com maior frequência foram seminários entre $60,4 \%$ (119) dos entrevistados e 57,4\% (113) aula expositiva; as atividades práticas vivenciadas mais relatadas foram: 40,1\% (79) palestras educativas e 35\% (69) feira de saúde (Tabela 2).

Tabela 2. Características acadêmicas de estudantes do curso de graduação em enfermagem, Salvador, Bahia, Brasil. 2017

\begin{tabular}{|c|c|c|}
\hline Variáveis & $n$ & $\%$ \\
\hline \multicolumn{3}{|l|}{ Tempo dedicado ao curso fora de sala de aula } \\
\hline$<1 \mathrm{~h}$ & 1 & 0,5 \\
\hline $1-2$ hs & 39 & 19,8 \\
\hline $3-4$ hs & 71 & 36,0 \\
\hline$>4$ hs & 64 & 32,5 \\
\hline \multicolumn{3}{|l|}{ Preferência do local para estudos* } \\
\hline Domicílio & 168 & 85,3 \\
\hline No curso & 64 & 32,5 \\
\hline Biblioteca & 55 & 27,9 \\
\hline Trabalho & 41 & 20,8 \\
\hline \multicolumn{3}{|l|}{ Material utilizado para estudos* } \\
\hline Site / Internet & 180 & 91,4 \\
\hline Livros & 148 & 75,1 \\
\hline Periódicos & 14 & 7,1 \\
\hline Outros & 4 & 2,0 \\
\hline \multicolumn{3}{|l|}{ Preferência em grupo de estudos* } \\
\hline Estudo individual & 143 & 72,6 \\
\hline Estudo em grupo & 61 & 31,0 \\
\hline Estudo em dupla & 42 & 21,3 \\
\hline \multicolumn{3}{|l|}{ Técnicas de ensino vivenciadas* } \\
\hline Seminário & 119 & 60,4 \\
\hline Aula expositiva & 113 & 57,4 \\
\hline Discussão em grupo & 98 & 49,7 \\
\hline Dinâmica de grupo & 71 & 36,0 \\
\hline Painel & 20 & 10,2 \\
\hline Oficina de trabalho & 14 & 7,1 \\
\hline \multicolumn{3}{|l|}{ Atividades práticas vivenciadas* } \\
\hline Palestras educativas na sala de espera & 79 & 40,1 \\
\hline Feira de saúde & 69 & 35,0 \\
\hline Assistência à saúde nos serviços & 55 & 27,9 \\
\hline Visita técnica com elaboração de relatório & 48 & 24,4 \\
\hline Participação em educação continuada & 43 & 21,8 \\
\hline
\end{tabular}

* Mais de uma alternativa de resposta para cada pergunta

Quanto à trajetória escolar pregressa, 77,7\% (153) estudaram na capital Salvador e 78,9\% (155) na rede pública. As justificativas pela escolha do curso de enfermagem foram variadas, sendo predominante a afinidade com área de saúde, com $62,4 \%$ (123).

Em relação à área de atuação almejada como futuro profissional enfermeiro: 38,6\% (76) indicaram a enfermagem obstétrica e 34,5\% (68) centro cirúrgico. Em se tratando da função do enfermeiro almejada pelo futuro profissional, a maior proporção dos entrevistados preferia a função assistencial com 73,6\% (145), seguida de função gerencial 43,1\% (85) (Tabela3). 
Tabela 3. Trajetória escolar pregressa, natureza do curso de ensino médio, justificativa pela escolha do curso de enfermagem e área de atuação profissional almejada por estudantes do curso de graduação em enfermagem, Salvador, Bahia, Brasil. 2017

\begin{tabular}{|c|c|c|}
\hline Variáveis & $\mathrm{n}$ & $\%$ \\
\hline \multicolumn{3}{|l|}{ Trajetória escolar pregressa } \\
\hline Capital & 153 & 77,7 \\
\hline Interior & 36 & 18,3 \\
\hline Outro Estado & 7 & 3,5 \\
\hline \multicolumn{3}{|l|}{ Natureza do Curso de ensino médio } \\
\hline Público & 155 & 78,9 \\
\hline Privado & 89 & 19,8 \\
\hline \multicolumn{3}{|l|}{ Justificativa pela escolha do curso de enfermagem } \\
\hline Afinidade com a área de saúde/ gostar de cuidar & 123 & 62,4 \\
\hline Mercado de trabalho promissor & 32 & 16,2 \\
\hline Vontade de ingressar no curso superior & 22 & 11,2 \\
\hline Já atua na área de saúde & 20 & 10,2 \\
\hline \multicolumn{3}{|l|}{ Área de atuação almejada como futuro profissional* } \\
\hline Enfermagem obstétrica & 76 & 38,6 \\
\hline Centro cirúrgico & 68 & 34,5 \\
\hline Cuidado ao paciente em estado crítico & 58 & 29,4 \\
\hline Enfermagem pediátrica & 55 & 27,9 \\
\hline Enfermagem geriátrica & 29 & 14,7 \\
\hline Saúde coletiva & 24 & 12,2 \\
\hline Saúde do trabalhador & 22 & 11,2 \\
\hline Saúde mental & 17 & 8,6 \\
\hline Outras & 18 & 9,1 \\
\hline \multicolumn{3}{|l|}{ Funções de enfermeiro almejadas* } \\
\hline Função assistencial & 145 & 73,6 \\
\hline Função gerencial & 85 & 43,1 \\
\hline Controle de risco com outros profissionais & 30 & 15,2 \\
\hline Função de ensino & 28 & 14,2 \\
\hline
\end{tabular}

$\left.{ }^{\star}\right)$ Mais de uma alternativa de resposta para cada pergunta

\section{Discussão}

No presente estudo observou o predomínio do sexo feminino entre estudantes no curso de graduação em enfermagem. Este resultado é consonante com outros estudos realizados na área sobre a profissão de enfermagem. ${ }^{2,6}$ Este dado tem sido prevalente em estudos com esse público devido à profissão da enfermagem ser, historicamente, predominante feminina, apesar do aumento registrado do sexo masculino de forma gradual. ${ }^{\mathrm{t}}$

No que se refere à faixa etária deste estudo, o curso é formado por estudantes jovens na faixa de 20 a 30 anos, em sua maioria, solteiros e sem filhos. Esses resultados se assemelham às características sociodemográficas dos estudos realizados em outras regiões do país ${ }^{6-7}$, porém, apresenta divergência ao constatar que $44,7 \%$ dos entrevistados responderam que possuem filhos e $23,4 \%$ pertenciam à faixa de 30 a 40 anos de idade. Este fato demonstra que o perfil dos estudantes universitários de enfermagem, embora seja de pessoas mais jovens, pessoas adultas com mais idade cada vez mais vêm se inserindo no ensino superior.

Verifica-se que estudantes de enfermagem que buscavam a formação na rede de ensino privado apresentam um perfil diferenciado dos estudantes das universidades públicas, corroborando estudos desenvolvidos em outras regiões do país. .-8 $^{-8}$ Outro aspecto relevante é que quase metade da população estudada já trabalha, tem filhos, mora distante da instituição de ensino e se locomove de transporte coletivo. Este dado demonstra que, durante a formação profissional, há necessidade em considerar esses fatores, assim como o desgaste físico decorrente do tempo gasto em locomoção para trabalho ou para a faculdade.

Para os estudantes, o local de estudo preferencial fora da sala de aula é o domicílio, de forma autônoma. Esta relação de preferência dos acadêmicos ao estudo individual em detrimento ao estudo em grupo, destaca-se, pois, a capacidade de dialogar com outros e trabalhar em equipe de forma interdisciplinar, é uma das competências exigidas para os profissionais atuais e do futuro, que visa alcance de objetivos comuns. Esta capacidade deve ser estimulada desde a época estudantil de várias maneiras na formação de enfermeiros, considerando a função primordial do profissional que implica em gestão de pessoas com manutenção de relacionamento interpessoal eficiente. ${ }^{9}$ 
Destaca-se que na formação profissional do enfermeiro, que se baseia na abordagem pedagógica que reorienta e qualifica, deve ser respaldada na prática profissional, a partir dos conhecimentos, das experiências e expectativas do sujeito em formação como a base do processo de ensino e aprendizagem no âmbito da formação profissional. 10

Por conseguinte, o uso de metodologias ativas que possam estimular a aprendizagem do estudante, possa também contribuir na formação do profissional enfermeiro competente nas dimensões, envolvidos em aspectos técnico, científico, político e eticamente aceitáveis e está cada vez mais considerado como elemento fundamental na aprendizagem significativa e valorizado nas práticas educativas. Acredita-se que esta aprendizagem reflexiva possa ajudar o estudante a se tornar responsável pela sua própria aprendizagem e ter autonomia na busca pelo conhecimento pertinente. ${ }^{11}$ Entretanto, na resposta dos entrevistados, ainda predominam a prática em sala de aula de transmissão de conhecimentos através de aulas expositivas ou recursos de seminário desenvolvido pelos estudantes e professores.

O reconhecimento por parte dos estudantes, da importância da integração entre estudantes, profissionais de saúde e comunidade em busca de ações interdisciplinares de trabalho e inter-relação dos envolvidos torna-se fundamental. E como aprendizagem, há necessidade de reflexão acerca do trabaIho em equipe de saúde que enfatize sua potência formativa, de integralidade na atuação, de democratização das relações de trabalho e responsabilidade enquanto profissional no período correspondente à formação do enfermeiro. 12

Os dados obtidos no estudo demonstram uma preferência dos estudantes pela área de atuação em enfermagem obstétrica. Apesar da necessidade de continuar investindo na área de atenção à saúde da criança e da mulher, o perfil epidemiológico da população brasileira e as demandas regionais apontam outras necessidades além dessa área. A característica regional da saúde destaca um crescimento da população idosa, das doenças crônicas, degenerativas e neoplasias, que aponta necessidade de investimento na formação de profissionais dotados de conhecimentos ampliados de saúde, capazes de trabalhar em promoção da saúde, prevenção das doenças e recuperação da saúde, integrados com outros profissionais de vários setores para o enfrentamento das demandas sociais. ${ }^{13}$

Desse modo, espera-se que o profissional reúna competência técnica, científica e tenha uma visão mais ampliada e integral do homem de modo a desempenhar sua atribuição como profissional com valores éticos e seja capaz de identificar as suas necessidades e buscar aprendizagem constante. Esta exigência ultrapassa a busca pela titulação do nível superior e objetiva a formação de um profissional crítico e reflexivo, dotado de uma visão mais ampla da sociedade capaz de atuar no interior dos estabelecimentos, sejam eles de atenção primária, secundária ou terciária. $\frac{14}{}$

Estudos realizados em outras regiões do país sobre a profissão enfermagem e o mercado de trabalho, apontam o desconhecimento dos jovens e adultos sobre a atuação do enfermeiro, suas potencialidades, oportunidades e dificuldades que envolvem este profissional no mercado de trabalho. O enfermeiro é considerado como o profissional que cuida dos doentes, e este cuidado é no âmbito assistencial, predominantemente, sem relatar o aspecto gerencial do trabalho de enfermagem, do ensino e do controle de risco na atuação interdisciplinar com outros profissionais. As autoras identificaram lacunas apontadas pelos entrevistados durante a trajetória acadêmica e o atual mercado de trabalho de enfermagem, consideram a importância da aproximação à realidade do campo profissional durante o curso através da integração teoria prática no seu desenvolvimento de forma gradual e abrangente. $\frac{15-16}{16}$

As pesquisas recentes sobre o mercado de trabalho de enfermagem e a inserção dos egressos no trabaIho apontam como elementos facilitadores no processo: a capacidade de liderança, gestão de pessoas, relações interpessoais e o preparo para docência durante a graduação de modo a desenvolver atuação em áreas de gerenciamento, de assistência, de pesquisa e de ensino. ${ }^{17}$ Desse modo, o resultado do estudo indica a necessidade de discutir com estudantes, durante a realização do curso, os problemas regionais de saúde e de competências necessárias do profissional para o enfrentamento da situação de cada momento. 
Este estudo apresenta limitações, como o fato de ter sido realizado apenas em uma IES de natureza privada e a pouca participação de estudantes dos dois últimos períodos do curso de enfermagem. Isso poderia ter influenciado na identificação de diferentes características sociodemográficas e acadêmicas devido ao perfil de outras IES e por estudantes formandos terem maior vivência na graduação. Espera-se que os estudantes de graduação, quanto mais próximo da conclusão do curso, mais expectativas eles têm com relação à sua formação profissional.

\section{Conclusão}

Os resultados apontaram que, entre as características sociodemográficas e acadêmicas dos estudantes de enfermagem, houve predomínio de pessoas do sexo feminino, de 20 a 30 anos de idade, solteiras, que moram distante da faculdade e utilizam transporte público para se deslocar para a IES, utilizam a internet como recurso para os estudos, e almejam atuar na assistência na área da enfermagem obstétrica após formação profissional.

Tão logo, existe a necessidade de estimular o desenvolvimento de habilidades e competências de estudantes de enfermagem durante a graduação para o trabalho em equipe e enfrentamento de desafios decorrentes do trabalho na área da saúde, o que pode ser alcançado a partir da interdisciplinaridade na formação e contato direto com os serviços de saúde e população. Para tanto, uma possibilidade seria adoção de atividades pedagógicas com metodologias ativas e em grupo na consecução de objetivos em comum. Exercitando, assim, a formação de um profissional reflexivo e crítico, capaz de atuar na realidade de saúde e de demanda.

Acredita-se que, apesar das limitações mencionadas, os resultados obtidos no estudo servem de subsídios na reformulação e atualização do Projeto Pedagógico do Curso (PPC) ora em execução na instituição e que contribuam na formação do profissional enfermeiro crítico, reflexivo e competente no exercício da sua profissão.

\section{Contribuições das autoras}

Saho M, Lomanto GA e Salviano ICB participaram da concepção e delineamento do estudo, redação e revisão do conteúdo intelectual, coleta e análise de dados até a versão final do manuscrito. Reis ES, Anjos KF e Rosa DOS participaram da redação e revisão do conteúdo intelectual até a versão final do manuscrito.

\section{Conflito de interesses}

Nenhum conflito financeiro, legal ou político envolvendo terceiros (governo, empresas e fundações privadas, etc.) foi declarado para nenhum aspecto do trabalho submetido (incluindo, mas não se limitando a subvenções e financiamentos, participação em conselho consultivo, desenho de estudo, preparação de manuscrito, análise estatística, etc.).

\section{Referências}

1. Resolução CNE/CES 3/2001, de 7 de novembro de 2001 (Brasil). Institui Diretrizes Curriculares do Curso de Graduação em Enfermagem. [Internet]. 2001 nov 7 [citado em 2016 out 10]. Disponível em: http://portal.mec.gov.br/cne/arquivos/pdf/CES03. pdf

2. Frota MA, Wermelinger MCMW, Vieira LJES, Ximenes Neto FRG, Queiroz RSM, Amorim RF. Mapeando a formação do enfermeiro no Brasil: desafios para atuação em cenários complexos e globalizados. Ciênc. saúde coletiva. 2020;25(1):25-35. https://doi. org/10.1590/1413-81232020251.27672019

3. Magnago C, Pierantoni CR. A formação de enfermeiros e sua aproximação com os pressupostos das Diretrizes Curriculares Nacionais e da Atenção Básica. Ciênc. saúde coletiva. 2020;25(1):15-24. https://doi.org/10.1590/1413$\underline{81232020251.28372019}$

\section{Marques LMNS. As metodologias ativas como estratégias para desenvolver a educação em valores na graduação em enfermagem. Esc Anna Nery. 2018;22(3):1-6. https://doi. org/10.1590/2177-9465-EAN-2018-0023}

5. Makuch DMV, Zagonel IPS. Abordagem pedagógica na implementação de programas curriculares na formação do enfermeiro. Esc Anna Nery. 2017;21(4):e20170025. https://doi. org/10.1590/2177-9465-EAN-2017-0025

6. Bublitz S, Guido LA, Kirchhof RS, Neves ET, Lopes LFD. Perfil sociodemográfico e acadêmico de discentes de enfermagem de quatro instituições brasileiras. Rev Gaúcha Enferm. 2015;36(1):7783. https://doi.org/10.1590/1983-1447.2015.01.48836 
7. Ximenes Neto FRG, Muniz CFF, Dias LJLF, Santos FD, Silva MAM, Oliveira EN. Perfil sociodemográfico dos estudantes de enfermagem da Universidade Estadual Vale do Acaraú (UVA). Enferm Foco. 2017; 8(3):75-9. https://doi.org/10.21675/2357707X.2017.v8.n3.1532

8. Colenci R, Berti HW. Formação profissional e inserção no mercado de trabalho: percepções de egresso de graduação em enfermagem. Rev esc enferm USP. 2012;46(1):158 -66. https://doi. org/10.1590/S0080-62342012000100022

9. Leonello NM, Vieira MPM, Duarte TCR. Competências para ação educativa de enfermeiras da estratégia de saúde da família. Rev Bras Enferm. 2018;71(3):1072-8. https://doi.org/10.1590/0034$\underline{7167-2017-0390}$

10. Siqueira MCG, Leopardi MT. O processo ensino-aprendizagem na formação de trabalhadores do SUS: reflexões a partir da experiência ETSUS. Trab educ saúde. 2016;14(1):114-36. https:// doi.org/10.1590/1981-7746-sip00094

11. Amaral APS, Boery RNSO, Vilela ABA, Sena ELS. Metodologias ativas: relato de experiência da participação em curso de especialização na área da saúde. Rev Docência Ens Sup. 2021;11:1-20. https://doi.org/10.35699/2237-5864.2021.24129

12. Costa LS, Formoso GA. Representações sociais de graduandos acerca do programa educação pelo trabalho para saúde. Rev Bras Enferm. 2018;71(2):244-51. https://doi.org/10.1590/0034-71672015-0168
13. Prefeitura Municipal de Salvador (Brasil), Secretaria da Saúde. Plano Municipal de Saúde 2018 - 2021 [Internet]. Salvador: Prefeitura de Salvador; 2018. Disponível em: http://www.saude. salvador.ba.gov.br/secretaria/informativos-e-documentacoes/

14. Nascimento PN, Barbosa MCL. Perspectivas dos graduandos de enfermagem frente ao mercado de trabalho. Id on Line Rev Psic [Internet]. 2017;11(35). Disponível em: https://idonline. emnuvens.com.br/id/article/view/730/0

15. Machado $\mathrm{MH}$, Koster I, Aguiar Filho W, Wermelinger MCMW, Freire NP, Pereira EJ. Mercado de trabalho e processos regulatórios - a Enfermagem no Brasil. Ciênc. saúde coletiva. 2020;25(1):101-12. https://doi.org/10.1590/1413$\underline{81232020251.27552019}$

16. Oliveira ISA, Pires DEP, Alvarez AM, Medeiros SM, Andrade SR. Tendências do mercado de trabalho de enfermeiros/as na visão de gestores. Rev Bras Enferm. 2018;71(1):148-55. https://doi. org/10.1590/0034-7167-2016-0103

17. Arcêncio RA. A enfermagem como profissão do futuro e base de sustentação dos sistemas universais. Rev. Latino-Am. Enfermagem. 2018;26:e3063. https://doi.org/10.1590/1518$\underline{8345.0000 .3063}$ 